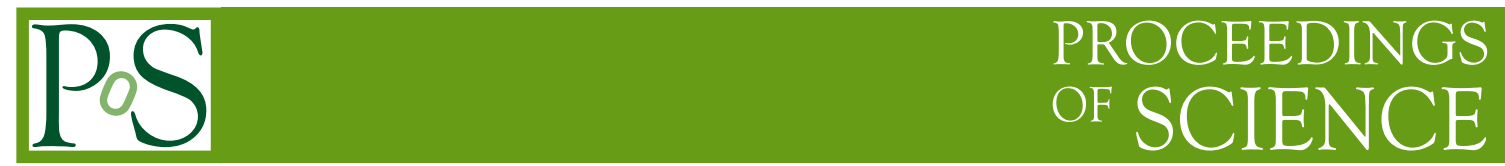

\title{
(In)direct search for SUSY at LHCb
}

\author{
Frédéric Machefert ${ }^{*}$ \\ LAL, Univ. Paris-Sud, Université Paris-Saclay, CNRS/IN2P3, Orsay, France. \\ E-mail: frederic.machefertein2p3.fr
}

The LHCb experiment is well adapted to indirect searches of new physics and more specifically to some SuperSymmetric signatures. In this paper, some studies performed by the $\mathrm{LHCb}$ collaboration on such signatures are presented, involving a $B_{(s)}^{0}$ meson decay in rare channels like $B_{(s)}^{0} \rightarrow \mu^{+} \mu^{-}$and $B_{(s)}^{0} \rightarrow \tau^{+} \tau^{-}$processes, in radiative decays with $b \rightarrow s \gamma$ transitions, or leading to a four-muon final state. The recently confirmed $\Sigma^{+}$decay in $p \mu^{+} \mu^{-}$is mentioned and the results of the search for an intermediate resonance are also given. Finally, some long lived and charged massive particle searches are described.

Sixth Annual Conference on Large Hadron Collider Physics (LHCP2018)

4-9 June 2018

Bologna, Italy

* Speaker.

${ }^{\dagger}$ On behalf of the LHCb collaboration. 


\section{Introduction}

The LHCb experiment [1] is one of the four large experiments of the LHC proton-proton collider at CERN (Geneva). It performes complementary (mostly indirect) searches for SuperSymmetry (SUSY) to the general purpose detectors ATLAS and CMS and benefits from some well-adapted features: an excellent vertex reconstruction (with a resolution of $\sigma_{\mathrm{PV}(\mathrm{z})}=71 \mu \mathrm{m}$ and $\sigma_{\mathrm{PV}(\mathrm{T})}=13 \mu \mathrm{m}$ for a 25 track primary vertex), a mass resolution of $0.5 \%$ (in $\mu \mu$ reconstruction), a particle identification for muons reaching $97 \%$ (with a mis-id of 1 to $3 \%$ in $\pi \rightarrow \mu$ ), a flexible trigger and low pile-up conditions.

\section{2. $B^{0} \rightarrow \mu^{+} \mu^{-}$and $B_{s}^{0} \rightarrow \mu^{+} \mu^{-}$very rare decays}

In the Standard Model (SM), the $B^{0} \rightarrow \mu^{+} \mu^{-}$and $B_{s}^{0} \rightarrow \mu^{+} \mu^{-}$processes only occur via flavour changing neutral currents and are further suppressed by helicity and CKM constraints. They are described by the effective field theory with an operator expansion containing the SM, a scalar and a pseudo-scalar contributions, the last two being no longer helicity-suppressed. SM predictions for these decays are $\mathscr{B}\left(B^{0} \rightarrow \mu^{+} \mu^{-}\right)=(1.06 \pm 0.09) \times 10^{-10}$ and $\mathscr{B}\left(B_{s}^{0} \rightarrow \mu^{+} \mu^{-}\right)=$ $(3.65 \pm 0.23) \times 10^{-9}$ [2]. The updated analysis performed by the LHCb collaboration includes a data sample of $4.4 \mathrm{fb}^{-1}$ collected during the runs 1 and 2 of the accelerator and uses some specific normalization channels $\left(B^{+} \rightarrow J / \psi K^{+}\right.$and $\left.B^{0} \rightarrow K^{+} \pi^{-}\right)$and a new selection based on a boosted decision tree trained on the simulations but calibrated on the data that improves the $B \rightarrow h^{+} h^{-}$ background rejection.
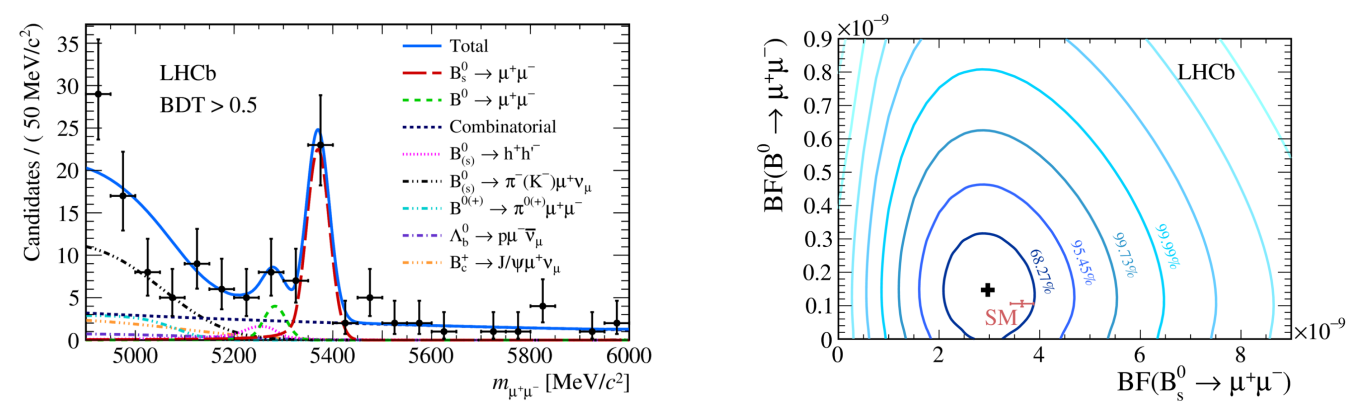

Figure 1: Left, fitted $\mu^{+} \mu^{-}$mass reconstruction for the $B_{(s)}^{0} \rightarrow \mu^{+} \mu^{-}$analysis with the signal and background contributions. Right, the combined $B^{0} \rightarrow \mu^{+} \mu^{-}$and $B_{s}^{0} \rightarrow \mu^{+} \mu^{-}$measurements with the SM expectation.

LHCb achieved the first "single experiment" observation of the $B_{s}^{0} \rightarrow \mu^{+} \mu^{-}$decay, and measured $\mathscr{B}\left(B^{0} \rightarrow \mu^{+} \mu^{-}\right)=\left(1.5_{-1.0-0.1}^{+1.2+0.2}\right) \times 10^{-10}$ at $1.6 \sigma$ (limit of $\mathscr{B}\left(B^{0} \rightarrow \mu^{+} \mu^{-}\right) \leq 3.4 \times 10^{-10}$ at $95 \%$ CL) and $\mathscr{B}\left(B_{s}^{0} \rightarrow \mu^{+} \mu^{-}\right)=\left(3.0 \pm 0.6_{-0.2}^{+0.3}\right) \times 10^{-9}$ at $7.8 \sigma$ [2] (see Fig. 1). In the SM, only the heavy $B_{s}^{0}$ mass eigenstate may decay via the $B_{s}^{0} \rightarrow \mu^{+} \mu^{-}$channel. Hence, the asymmetry rate

$$
A_{\Delta \Gamma}=\frac{\Gamma\left(B_{s}^{H} \rightarrow \mu^{+} \mu^{-}\right)-\Gamma\left(B_{s}^{L} \rightarrow \mu^{+} \mu^{-}\right)}{\Gamma\left(B_{s}^{H} \rightarrow \mu^{+} \mu^{-}\right)+\Gamma\left(B_{s}^{L} \rightarrow \mu^{+} \mu^{-}\right)}
$$



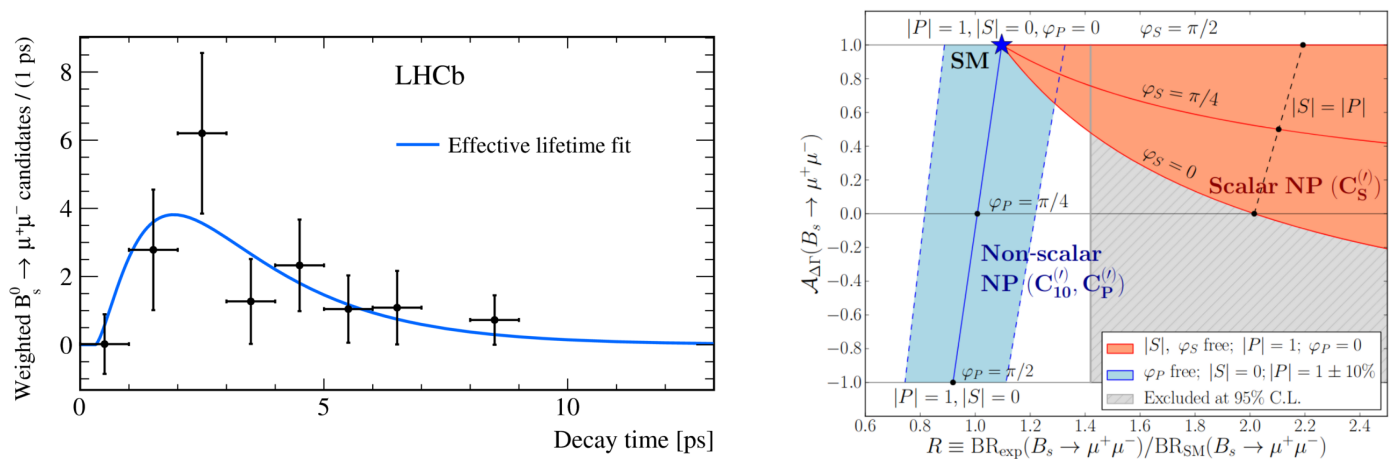

Figure 2: Left, $B_{s}^{0} \rightarrow \mu^{+} \mu^{-}$decay time and effective lifetime $\tau\left(\mu^{+} \mu^{-}\right)$fit. Right, dependence of the asymmetry rate $A_{\Delta \Gamma}$ with the SM and scalar and pseudo-scalar new physics contributions [3].

takes the value +1 in the SM. It may be determined by measuring the effective lifetime $\tau\left(\mu^{+} \mu^{-}\right)$ (see Fig. 2, left), and is an observable complementary to the branching ratio in the search for new physics. Figure 2 (right) shows the dependence of the $A_{\Delta \Gamma}$ asymmetry rate with respect to the SM $\left(C_{10}^{(\prime)}\right)$, scalar $\left(C_{S}^{(\prime)}\right)$ and pseudo-scalar $\left(C_{P}^{(\prime)}\right)$ new physics contributions to the operator expansion [3, 4].

The first $\tau\left(\mu^{+} \mu^{-}\right)$effective lifetime measurement using the run 1 and 2 data [2] is $\tau\left(\mu^{+} \mu^{-}\right)=$ $2.04 \pm 0.44 \pm 0.05 \mathrm{ps}$, consistent with the SM prediction, and leading to a determination of $A_{\Delta \Gamma}$ within $1 \sigma$ of $A_{\Delta \Gamma}=+1$ and $1.4 \sigma$ of $A_{\Delta \Gamma}=-1$.

\section{3. $B^{0} \rightarrow \tau^{+} \tau^{-}$and $B_{s}^{0} \rightarrow \tau^{+} \tau^{-}$decays}

Similarly to the $\mu^{+} \mu^{-}$final state, the $B^{0} \rightarrow \tau^{+} \tau^{-}$and $B_{s}^{0} \rightarrow \tau^{+} \tau^{-}$decays are studied by the LHCb collaboration. In spite of a helicity suppression effect and a sensitivity to new physics which are reduced, this mode will provide a lepton flavour violation test altogether with the $\mu^{+} \mu^{-}$ channels. A precise SM prediction [5] exists leading to $\mathscr{B}\left(B^{0} \rightarrow \tau^{+} \tau^{-}\right)=(2.22 \pm 0.19) \times 10^{-9}$ and $\mathscr{B}\left(B^{0} \rightarrow \tau^{+} \tau^{-}\right)=(7.73 \pm 0.49) \times 10^{-10}$. The LHCb analysis is based on $3 \mathrm{fb}^{-1}$ and uses hadronic tau decays. The limits obtained are $\mathscr{B}\left(B^{0} \rightarrow \tau^{+} \tau^{-}\right)<2.1 \times 10^{-3}$ and $\mathscr{B}\left(B^{0} \rightarrow \tau^{+} \tau^{-}\right)<6.8 \times 10^{-3}$ at $95 \%$ CL [6].

\section{Radiative b decays}

Radiative decays are $b \rightarrow s \gamma$ transitions which are described by an effective field theory and the operator expansion

$$
H_{e f f}=\frac{-4 G_{G}}{\sqrt{2}} V_{t s}^{\star} V_{t b}\left(C_{7} O_{7}+C_{10}^{\left({ }^{\prime}\right)} O_{10}^{(\prime)}\right)
$$

In the SM, the photon is predominantly left-handed, the right-handed contribution being heavily suppressed by a factor $m_{s} / m_{b}$. Potentially, new physics may enhance the right-handed contribution, especially with contributions of supersymmetric partners and charged Higgs bosons.

Radiative b decays have been first observed in the $B^{+} \rightarrow K^{+} \pi^{+} \pi^{-}$decay channel at LHCb [7] and have been studied through the $B^{0} \rightarrow K^{\star 0} l^{+} l^{-}$decays at low $q^{2}\left(\sim m_{\mu \mu}^{2}\right)$ [8]. 


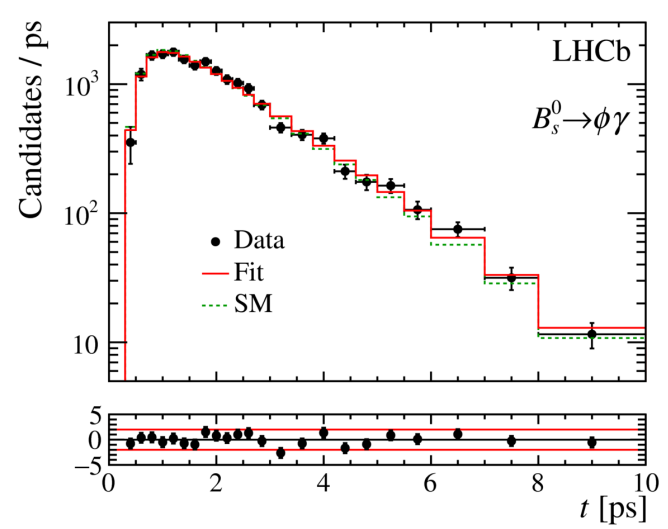

Figure 3: $B_{s} \rightarrow \phi \gamma$ decay time measurement. The SM expectation is given by the green dotted histogram, the fit to the data (dots) is in red.

The photon polarisation can be measured in the $B_{s} \rightarrow \phi \gamma$ channel from the time-dependent decay rate

$$
\Gamma_{B_{s}+\overline{B_{s}}(t)} \simeq e^{-\Gamma_{s} t}\left[\cosh \left(\frac{\Delta \Gamma_{s} t}{2}\right)-A^{\Delta} \sinh \left(\frac{\Delta \Gamma_{s} t}{2}\right)\right]
$$

where $A^{\Delta}$ is related to the left and right handed amplitudes and therefore to $C_{7}$. In the SM, $A^{\Delta}=$ $0.047_{-0.025}^{+0.029}$. The first measurement of the photon polarisation in $B_{S}$ decay [9], obtained in the channel $B_{s} \rightarrow \phi\left(K^{+} K^{-}\right) \gamma$ from a data sample of $3 \mathrm{fb}^{-1}$ is $A^{\Delta}=-0.98_{-0.52-0.20}^{+0.46+0.23}$, which is consistent with the SM predictions. Figure 3 shows the decay time fit, the corresponding data and the SM expectations.

\section{5. $\Sigma^{+}$decay in $p \mu^{+} \mu^{-}$}

The HyperCP collaboration has observed the decay $\Sigma^{+} \rightarrow p \mu^{+} \mu^{-}$. The branching ratio measured [10] is $\left(8.6_{-5.4}^{+6.6} \pm 5.5\right) \times 10^{-8}$, which is consistent with the SM predictions [11]. Three events observed for this measurement had a similar di-muon mass, which could be due to the fact that the decay proceeds via a resonance $X^{0}$, in $\Sigma^{+} \rightarrow p X^{0}\left(\mu^{+} \mu^{-}\right)$.

The decay $\Sigma^{+} \rightarrow p \mu^{+} \mu^{-}$has been confirmed by LHCb with $3 \mathrm{fb}^{1}$ of data, with a branching ratio of $\left(2.2_{-1.3}^{+1.8}\right) \times 10^{-8}$ and 13 events observed leading to a significance of $4.1 \sigma$ [12]. However, no evidence for a di-muon resonance at $214 \mathrm{MeV} / \mathrm{c}^{2}$ has been found (see Fig. 4).

\section{6. $B_{(s)}^{0}$ decay in four muons}

The decay of the $B^{0}(s)$ mesons into four muons is strongly suppressed in the SM. However, il may proceed either through resonant or non-resonant modes. New physics may enhance the decay rates up to $10^{-4}$. For example, the MSSM allows the mode $B^{0}(s) \rightarrow P\left(\mu^{+} \mu^{-}\right) S\left(\mu^{+} \mu^{-}\right)$where $S$ and $P$ are sgoldstino particles. 

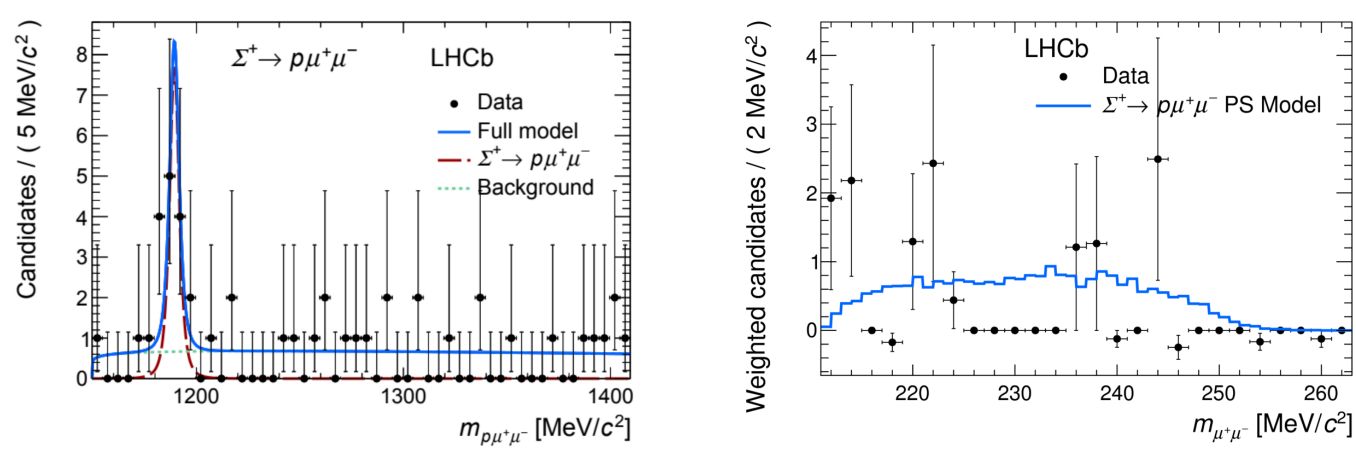

Figure 4: Left, $p \mu^{+} \mu^{-}$reconstructed mass showing the $\Sigma^{+}$resonance. Right, the corresponding $\mu^{+} \mu^{-}$ reconstructed mass exhibiting no specific structure.

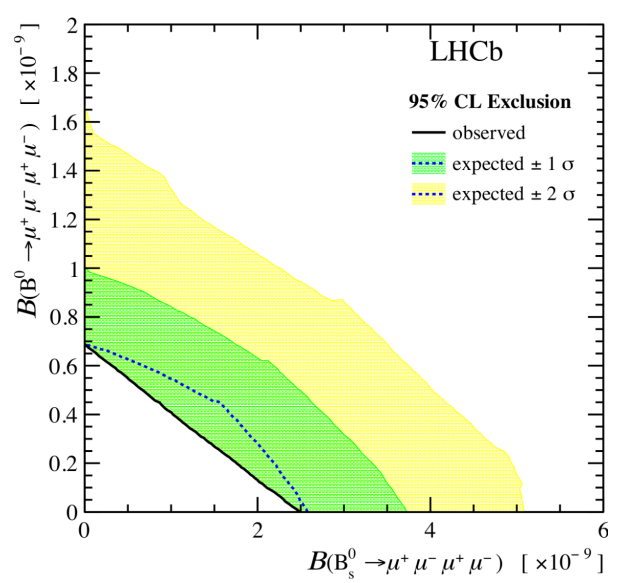

Figure 5: $\mathscr{B}\left(B^{0} \rightarrow \mu^{+} \mu^{-} \mu^{+} \mu^{-}\right)$and $\mathscr{B}\left(B_{s}^{0} \rightarrow \mu^{+} \mu^{-} \mu^{+} \mu^{-}\right)$exclusion limits obtained from the $2 \mathrm{fb}^{-1}$ data sample recorded by $\mathrm{LHCb}$.

A first analysis was performed with $2 \mathrm{fb}^{-1}$, but was recently updated with an extended data sample, an improved selection based on a multivariate classifier and a new normalisation mode. No event was observed, but the upper limits on the branching fraction were improved, leading to

$$
\begin{array}{ll}
\mathscr{B}\left(B^{0} \rightarrow \mu^{+} \mu^{-} \mu^{+} \mu^{-}\right) & <6.9 \times 10^{-8} \\
\mathscr{B}\left(B_{s}^{0} \rightarrow \mu^{+} \mu^{-} \mu^{+} \mu^{-}\right) & <2.5 \times 10^{-9} \\
\mathscr{B}\left(B^{0} \rightarrow S\left(\mu^{+} \mu^{-}\right) P\left(\mu^{+} \mu^{-}\right)\right) & <6.0 \times 10^{-10} \\
\mathscr{B}\left(B_{s}^{0} \rightarrow S\left(\mu^{+} \mu^{-}\right) P\left(\mu^{+} \mu^{-}\right)\right) & <2.2 \times 10^{-9}
\end{array}
$$

with the assumptions $m_{s}=2.6 \mathrm{GeV} / \mathrm{c}^{2}, m_{p}=214.3 \mathrm{MeV} / \mathrm{c}^{2}$, for the last two limits given [13].

\section{Search for long lived particles decaying semileptonically}

The long lived particles (LLP) can be produced either through B or D decays or in the pp collisions. The knowledge of the vertex region of the experiment is crucial in order to reduce the 
background due to the interaction of the particles with the vertex locator RF foil, in the example of LHCb. A p-value is assigned to the hypothesis that a secondary vertex originates from material interaction.
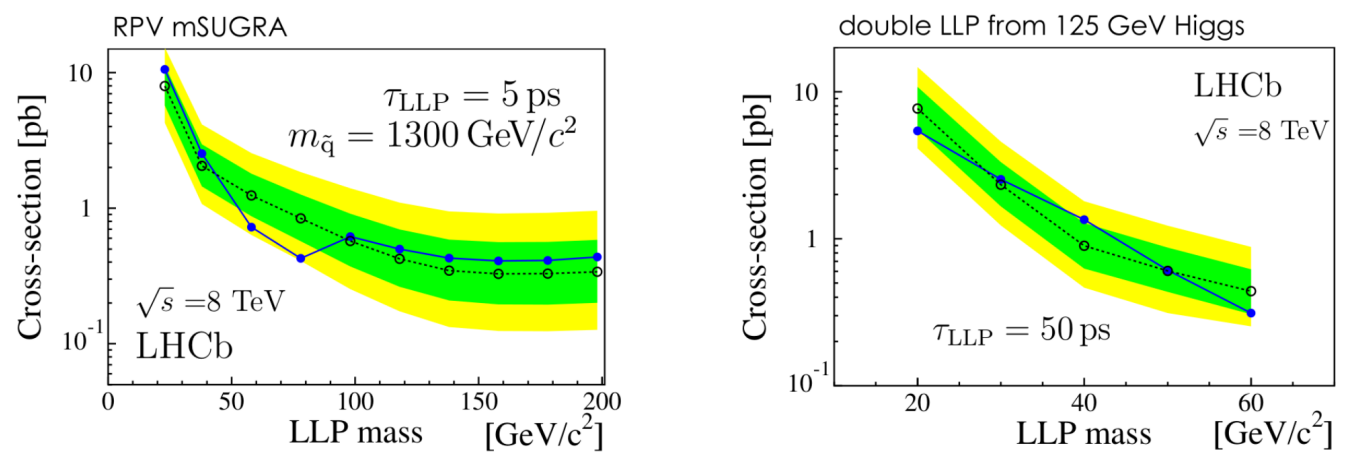

Figure 6: Expected (open dots with $1 \sigma$ and $2 \sigma$ bands) and observed (full dots) cross-section times branching fraction upper limits at 95\% confidence level, as a function of the LLP mass, in the RPV-mSUGRA (left) and simplified topologies (right) approaches [14].

Two approaches to search for massive LLP decaying semileptonically into SM particles have been followed: a RP violating mSUGRA neutralino type, in a mass range in $23-198 \mathrm{GeV} / \mathrm{c}^{2}$; less model-dependent simplified topologies in the mass range $25-80 \mathrm{GeV} / \mathrm{c}^{2}$. The run 1 dataset $\left(3 \mathrm{fb}^{-1}\right)$ was used, the trigger being based on a large $P_{\mathrm{T}}$ muon and a displaced vertex in the lifetime range $5-100$ ps. In the analysis a muon isolation criteria was applied. The main background was due to $b \bar{b}$ events and a signal yield was measured from a simultaneous fit of the LLP reconstructed mass in both the "high" and "low" isolation regions corresponding to the expected signal and background regions. The signal yield is compatible with zero events [14] (see Fig 6).

\section{Acknowledgements}

The author wishes to thank the organizers of the LHCP 2018 conference for their hospitality during the conference and the members of LHCb for their help.

\section{References}

[1] R. Aaij et al. (LHCb Collaboration), LHCb detector performance, Int. J. Mod. Phys. A 30, No. 07, $1530022(2015)$

[2] R. Aaij et al. (LHCb Collaboration), Measurement of the $B_{s}^{0} \rightarrow \mu^{+} \mu^{-}$Branching Fraction and Effective Lifetime and Search for $B^{0} \rightarrow \mu^{+} \mu^{-}$Decays, Phys. Rev. Lett. 118, 191801 (2017)

[3] K. De Bruyn et al., Probing New Physics via the $B_{s}^{0} \rightarrow \mu^{+} \mu^{-}$Effective Lifetime, Phys. Rev. Lett. 109, 041801 (2012)

[4] W. Altmannshofer, C. Niehoff, D. M. Straub, $B_{s}^{0} \rightarrow \mu^{+} \mu^{-}$as current and future probe of new physics, J. High Energy Phys. 05 (2017) 076

[5] C. Bobeth et al., $B_{s, d} \rightarrow l^{+} l^{-}$in the Standard Model with Reduced Theoretical Uncertainty, Phys. Rev. Lett. 112, 101801 (2014) 
[6] R. Aaij et al. (LHCb Collaboration), Search for the Decays $B_{s}^{0} \rightarrow \tau^{+} \tau^{-}$and $B^{0} \rightarrow \tau^{+} \tau^{-}$, Phys. Rev. Lett. 118, 251802 (2017)

[7] R. Aaij et al. (LHCb Collaboration), Observation of Photon Polarization in the $b \rightarrow \gamma$ Transition, Phys. Rev. Lett. 112, 161801 (2014)

[8] R. Aaij et al. (LHCb Collaboration), Angular analysis of the $B^{0} \rightarrow K^{\star} e^{+} e^{-}$decay in the low-q2 region, J. High Energy Phys. 04 (2015) 064

[9] R. Aaij et al. (LHCb Collaboration), First Experimental Study of Photon Polarization in Radiative $B_{s}^{0}$ Decays, Phys. Rev. Lett. 118, 021801 (2017)

[10] H. K. Park et al. (HyperCP Collaboration), Evidence for the Decay $\Sigma \rightarrow p \mu^{+} \mu^{-}$, Phys. Rev. Lett. 94, 021801 (2005)

[11] X.-G. He, J. Tandean, and G. Valencia, Decay $\Sigma \rightarrow p l^{+} l^{-}$within the standard model, Phys. Rev. D 72 , 074003 (2005)

[12] R. Aaij et al. (LHCb Collaboration), Evidence for the rare decay $\Sigma \rightarrow p \mu^{+} \mu^{-}$, Phys. Rev. Lett. 118, 221803 (2018)

[13] R. Aaij et al. (LHCb Collaboration), Search for decays of neutral beauty mesons into four muons, J. High Energy Phys. 03 (2017) 001

[14] R. Aaij et al. (LHCb Collaboration), Search for massive long-lived particles decaying semileptonically in the LHCb detector, Eur. Phys. J. C (2017) 77: 224 